\title{
Functional analysis of Spodoptera frugiperda nucleopolyhedrovirus late expression factors in Sf9 cells
}

\author{
Marcelo F. Berretta $\cdot$ M. Gabriela López \\ Oscar Taboga $\cdot$ Alicia Sciocco-Cap • \\ Víctor Romanowski
}

Received: 26 June 2012/ Accepted: 25 October 2012/Published online: 4 November 2012

(C) Springer Science+Business Media New York 2012

\begin{abstract}
We used transient expression assays to assess the function of the baculovirus Spodoptera frugiperda $\mathrm{M}$ nucleopolyhedrovirus (SfMNPV) homologs of Autographa californica MNPV (AcMNPV) factors involved in late gene expression (lefs), in the Sf9 insect cell-line, which is permissive for both viruses. It is well-established that nineteen AcMNPV lefs support optimal levels of activity from a late promoter-reporter gene cassette in this assay. A subgroup of SfMNPV lefs predicted to function in transcription-specific events substituted the corresponding AcMNPV lefs very efficiently. When all SfMNPV lefs were assayed, including replication lefs, activity was low, but addition of two AcMNPV lefs not encoded in SfMNPV genome, resulted in augmented reporter activity. SfMNPV IE-1 was able to activate an early promoter cis-linked to an $h r$-derived element from SfMNPV but not from AcMNPV. However, the level of early promoter activation with SfMNPV IE-1 was lower compared to AcMNPV IE-1.
\end{abstract}

Keywords Late expression factors - AcMNPV . SfMNPV

M. F. Berretta $(\bowtie) \cdot$ A. Sciocco-Cap

Instituto de Microbiología y Zoología Agrícola (IMYZA),

INTA Castelar, Castelar, Argentina

e-mail: mberretta@cnia.inta.gov.ar

M. G. López · O. Taboga

Instituto de Biotecnología (IB), INTA Castelar, Castelar,

Argentina

V. Romanowski

Instituto de Biotecnología y Biología Molecular (IBBM, CONICET-UNLP), Facultad de Ciencias Exactas, Universidad Nacional de La Plata, La Plata, Argentina

\section{Introduction}

Baculoviruses are large DNA viruses pathogenic to insects. At the late stage of infection, they produce distinct proteinaceous structures known as occlusion bodies (OBs) that embed the virions responsible for the horizontal transmission of the virus between susceptible larval hosts. According to the shape and size of OBs, baculoviruses were traditionally classified as nucleopolyhedroviruses (NPV) or granuloviruses (GV). A recent revision of the taxonomy of the family determined four groups at the genus level: Alphabaculovirus (lepidopteran NPV), Betabaculovirus (lepidopteran GV), Gammabaculovirus (hymenopteran NPV), and Deltabaculovirus (dipteran NPV) [1]. With around 60 baculovirus genomes sequenced to date, there is a critical volume of information to outline baculovirus diversity. Almost 900 different orthologous genes have been identified; in contrast, only 31 genes, referred to as core genes, are present in all genomes [2]. About one-third of core genes are genes related to DNA replication or processing and expression of late and very late genes. Most of our knowledge on baculovirus gene expression comes from studies on the type species Autographa californica $\mathrm{M}$ nucleopolyhedrovirus (AcMNPV), and a few other phylogenetically related species within group I of the alphabaculoviruses, as defined by Zanotto et al. [3]. Species belonging to group II of the alphabaculoviruses have more heterogeneous gene content and have been less studied with regard to molecular events of the viral infection cycle.

Baculoviruses express their genes in three temporally regulated phases: early, late, and very late. Transcription of early genes depends on the cellular RNA polymerase II. Expression of immediate-early (ie-) genes proceeds independently of viral factors, while expression of delayed early genes requires transactivation by other viral factors. IE-1 is a major transactivator of early gene expression in 
AcMNPV although at least two other ie- genes also activate early promoters [4]. IE-1-mediated transactivation of early promoters increases when IE-1 binds to enhancers known as homologous regions (hrs) [5]. Hrs also serve as origins of DNA replication (ori) in assays of virus-induced plasmid replication [6, 7]. Baculovirus late and very late genes are transcribed from the conserved promoter motif TAAG [8] by an RNA polymerase encoded by the virus [9, 10]. They are the only DNA viruses which replicate in the nuclei of cells that encode their own DNA-directed RNA polymerase. Transcription of late genes requires viral DNA replication; therefore, genes necessary for late expression, collectively known as late expression factors (lefs), include not only genes encoding the RNA polymerase subunits and some other factors that are not well characterized but also those encoding the replication machinery [11]. AcMNPV lefs were discovered using different approaches including marker rescue experiments with temperature-sensitive mutants, and transient expression assays conducted in the Spodoptera frugiperda-derived Sf21 cell-line (reviewed in [11]). In the latter, a plasmid containing a reporter gene driven by a late promoter, and an $h r$ sequence that functions as ori, was cotransfected into cells along with AcMNPV genomic fragments that were progressively shortened to eventually identify individual genes required for optimal reporter gene activity [12]. Nineteen AcMNPV lefs were defined in this manner [13] (Table 1), while nine of these genes were identified as replication factors in plasmid replication assays [14, 15]. Some lefs are implicated in determining host specificity in cell culture [16, 17]. The knock-out of several lefs has provided complementary information about their actual requirement in the context of the virus replication cycle [18].

Spodoptera frugiperda M nucleopolyhedrovirus (SfMNPV) is a member of group II alphabaculoviruses that has potential to be developed as biocontrol agent against the fall armyworm, $S$. frugiperda, an agricultural pest of economic importance in the Americas [19, 20]. SfMNPV is a host-specific virus, and in cell culture it infects productively the Sf21 cell-line [21-23] and its clonal derivative Sf9 [24]. Early studies of pulse-labeled proteins of SfMNPV-infected Sf21 cells, showed a program of protein synthesis throughout the infection cycle similar to that observed in this cell-line infected with AcMNPV [23, 25]. SfMNPV has been studied for many years although it was not until recently that the complete sequence of SfMNPV genomes became available [26-28]. Of the 19 lefs identified in AcMNPV, only 16 homologs were found in SfMNPV genome (Table 1). Being smaller in number, genes predicted as lefs may have functionally evolved in this species to fulfill late gene expression. Alternatively, other genes may affect this process in SfMNPV, either directly or indirectly. Likewise, in AcMNPV additional genes may be considered as lefs [11]. For baculovirus species belonging to group II alphabaculoviruses there are few studies aimed to characterize lefs involved in replication [29, 30], but there is a lack of experimental evidence with regard to the functionality of the complete set of genes homologous to AcMNPV lefs in a permissive cellular environment. In this study, we assessed the ability of SfMNPV lefs to support late gene expression in transient assays, in Sf9 cells. For this purpose, we adapted the system originally developed to study AcMNPV lefs, and found that only the subgroup of SfMNPV lefs corresponding to lefs considered involved in transcription-specific activities, substituted for their AcMNPV counterparts very efficiently, while SfMNPV replication lefs exhibited low-level functionality. Since $i e-1$ is included in the group of replication lefs, we further investigated its functionality in an early gene expression assay. Results presented here are discussed in terms of the utility of our system for screening SfMNPV genome for genes potentially influencing gene expression in this species and possibly in other group II alphabaculoviruses.

\section{Materials and methods}

\section{Cells and viruses}

Spodoptera frugiperda Sf9 cells obtained from American Type Culture Collection (ATCC) were cultured at $27{ }^{\circ} \mathrm{C}$ as a monolayer in Sf900 II insect medium (Invitrogen) supplemented with $1 \%$ fetal bovine serum (Internegocios, Argentina) and $1 \%$ antibiotic-antimycotic solution (GIBCO). The SfMNPV stock used in this study, SfMNPV-AR, was originally obtained from a single $S$. frugiperda larva and was characterized as a single isolate [31].

\section{Plasmid constructions}

Plasmids utilized to assess late gene expression contained luciferase as reporter gene under control of late promoter of AcMNPV vp39 capsid gene $c i s$-linked to a fragment of the specified $h r$ sequence. In reporter plasmid pAchr5CAPluc, the $h r$ fragment (AC $h r$ ) corresponds to AcMNPV $h r 5$ [32], and in pSf $h r l \mathrm{CAPluc}$, the $h r(\mathrm{Sf} h r$ ) corresponds to a fragment of SfMNPV-AR $h r l$ that encompasses five palindromic repeats. pSfhrlCAPluc was constructed in three steps. First, a 616-bp fragment of SfMNPV-AR $h r l$ was amplified with primers Fwd 5'-TTTTCATTAACTAACCACCATCA-3' and Rev 5'-CCCGGGGATTGCAAAGATTAACTGTAAC AAAC-3' (XmaI site underlined); the PCR product was cloned in vector $\mathrm{pCR}{ }^{\circledR} 4$-TOPO (Invitrogen), then removed by digestion with NotI and XmaI and gel-purified. In parallel, a reporter plasmid containing CAT under AcMNPV vp39 
Table 1 Comparison of AcMNPV and SfMNPV lefs

\begin{tabular}{|c|c|c|c|c|c|c|}
\hline \multirow[t]{2}{*}{ ORF name } & \multicolumn{2}{|c|}{ ORF number } & \multicolumn{2}{|c|}{ Amino acid residues } & \multirow{2}{*}{$\begin{array}{l}\text { Amino acid } \\
\text { identity }(\%)\end{array}$} & \multirow[t]{2}{*}{ AcMNPV LEF function } \\
\hline & AcMNPV & SfMNPV $^{\mathrm{a}}$ & AcMNPV & SfMNPV $^{\mathrm{a}}$ & & \\
\hline lef-1 & 14 & 18 & 266 & 239 & 40 & DNA primase \\
\hline lef-2 & 6 & 16 & 210 & 213 & 45 & Primase accessory protein \\
\hline lef-3 & 67 & 90 & 385 & 388 & 28 & ssDNA binding protein \\
\hline$l e f-4$ & 90 & 76 & 464 & 459 & 47 & $\begin{array}{l}\text { Capping enzyme, RNA polymerase } \\
\text { complex }\end{array}$ \\
\hline lef-5 & 99 & 68 & 265 & 277 & 54 & Transcription factor \\
\hline lef-6 & 28 & 128 & 173 & 155 & 32 & Transcription factor \\
\hline lef-7 & 125 & 21 & 226 & 351 & Not sig & Possible ssDNA binding protein \\
\hline lef-8 & 50 & 113 & 876 & 881 & 61 & RNA polymerase complex \\
\hline lef-9 & 62 & 97 & $490^{\mathrm{b}}$ & 498 & 65 & RNA polymerase complex \\
\hline lef-10 & $53 \mathrm{a}$ & 106 & 78 & 75 & 48 & Transcription factor \\
\hline lef-11 & 37 & 120 & 112 & 133 & 37 & Replication/transcription factor \\
\hline lef-12 & 41 & - & 181 & - & - & Transcription factor \\
\hline$i e-1$ & 147 & 136 & 582 & 681 & 31 & Transactivator, $h r$-binding protein \\
\hline$i e-2$ & 151 & - & 408 & - & - & Transactivator, cell-cycle control \\
\hline dnapol & 65 & 92 & 984 & 1,040 & 45 & DNA polymerase \\
\hline p143 & 95 & 72 & 1,221 & 1,228 & 41 & DNA helicase \\
\hline $39 K$ & 36 & 121 & 275 & 309 & 33 & Transcription factor \\
\hline$p 47$ & 40 & 116 & 401 & 399 & 54 & RNA polymerase complex \\
\hline p35 & 135 & - & 299 & - & - & Apoptosis inhibitor \\
\hline
\end{tabular}

ssDNA single-stranded DNA

a Values correspond to GenBank accession HM595733

b Value corresponds to the protein product translated from the second ATG of the ORF

promoter, pCAPCAT $\Delta \mathrm{HN} \Delta \mathrm{SE}[32]$, was digested with the same enzymes to separate Ac $h r$, and the resulting 4,740 bp backbone was ligated to Sf $h r$. Finally, this construct was digested with SacI and BglII, and the 1,187 bp fragment containing Sf $h r$ cis-linked to AcMNPV vp39 promoter was purified and ligated to the backbone of pAchr5CAPluc previously treated with the same enzymes to remove the tandem Ac $h r$-vp39 promoter.

The construction of the AcMNPV lef library in plasmid $\mathrm{pHSEpiHisVI}^{+}$has been described [13]. In this vector, each lef was cloned under Drosophila heat shock protein (hsp) 70 promoter and fused to HA and His tags at the N-terminus. To clone the sixteen SfMNPV lefs individually in the same vector, we amplified by PCR each lef ORF from the second codon to the stop codon with specific forward and reverse primers listed in Table 2, and using SfMNPV-AR DNA as template. The PCR products were digested with $B g l \mathrm{II}$ and NotI sites (except for ie-1 and $p 47$, which were digested with BamHI and NotI) and cloned in the respective sites of pHSEpiHisVI ${ }^{+}$. All constructs were verified by nucleotide sequencing. Plasmid SfieOH-X consists in an XbaI-HindIII SfMNPV-AR genomic fragment of approximately 7,250 bp (see Fig. 1c) cloned in the respective sites of plasmid pcDNAII (Invitrogen), extending from 228 bp upstream of exon-0 ORF to 757 bp downstream of stop codon of ie-1 ORF. Sfie-1S-X is a subclone of SfieOH-X that was obtained by first digesting the latter with SacI and HindIII to remove exon-0 ORF; then, the linearized vector was bluntended and religated. The resulting clone retained ie-1 including $515 \mathrm{bp}$ upstream of the ORF and downstream sequence as in SfieOH-X.

Reporter plasmid pBAS35K-Luc/28mer-up+/PA has been described [33], and was used in this study to assess early gene expression. In this plasmid luciferase is driven by the TATA box-containing basal promoter of AcMNPV p35 gene (nucleotides -30 to +12 , where position +1 corresponds to the transcriptional start site) with a 28-mer imperfect palindromic repeat from AcMNPV cis-linked upstream to the basal promoter. The reporter plasmid pBAS35K-Luc/46mer contains a 46-mer imperfect palindrome corresponding to the third repeat of SfMNPV-AR $h r l$ that matches exactly the homologous repeats from two sequenced SfMNPV isolates (GenBank accession numbers EF035042 [27] and HM595733 [28]). This plasmid was constructed by first digesting pBAS35K-Luc/28mer-up+/ PA with $B g l I I$ and $M l u I$ to remove the 28-mer sequence between these restriction sites and then by ligation of the backbone to annealed oligonucleotides $5^{\prime}$-GATCTTCAAA 
Table 2 Oligonucleotides used for amplification of SfMNPV lefs

${ }^{a}$ Oligonucleotide name includes the corresponding lef name italicized and in boldtype. $\mathrm{N}$ and $\mathrm{C}$ denote forward and reverse oligonucleotide, respectively

b Restriction endonuclease recognition sites are underlined

\begin{tabular}{|c|c|}
\hline Oligonucleotide $^{\mathrm{a}}$ & Sequence $^{\mathrm{b}}$ \\
\hline Sflef1N-BglII & GAAGATCTGCATCATTCTTATCCTGCATTCA \\
\hline Sflef1C-NotI & GAATGCGGCCGCTTAAGATTTGGTAGTAGTTAGTAAATACGA \\
\hline Sflef $2 \mathrm{~N}-B g l \mathrm{II}$ & GAAGATCTGCGGCGGAACAGTTACTGTCGT \\
\hline Sflef2C-NotI & GAATGCGGCCGCTCAATAGTTACAAATAGGATTAGTTCCT \\
\hline Sflef3N-BglII & GCAGATCTTCTTTGTCTACGGAAATGCCTAC \\
\hline Sflef3C-NotI & GAATGCGGCCGCTTAAAAACCTTCAAAGTTATCAGT \\
\hline Sflef $4 \mathrm{~N}-B g l \mathrm{II}$ & GAAGATCTGTTGTCGAAAACGAAATTTCTTA \\
\hline Sflef4C-Not I & GAATGCGGCCGCTTAATTAGGCACCAAACGATCT \\
\hline Sflef $5 \mathrm{~N}-B g l \mathrm{II}$ & GCAGATCTTCTAAAGCTAACGCATGCCAAGT \\
\hline Sflef5C-NotI & GAATGCGGCCGCTTAGTTAACCGTGACGATGCGGT \\
\hline Sflef6N-BglII & GCAGATCTTATGTGTTTTATATTAACGGTACT \\
\hline Sflef6C-NotI & GAATGCGGCCGCTTATTTTTTATCATAAAGTTTATCA \\
\hline Sflef7N-BglII & GCAGATCTTGTGTTCGTGTAGTATATAAACGGT \\
\hline Sflef7C-NotI & GAATGCGGCCGCTTAATAATCATCAAAACTTCCCA \\
\hline Sflef $8 \mathrm{~N}-B g l \mathrm{II}$ & GAAGATCTACGGACGTGATTGTCGATTTTAA \\
\hline Sflef8C-NotI & GAATGCGGCCGCTTATCTCATAATTGTATTATTACAGT \\
\hline Sflef $9 \mathrm{~N}-B g l \mathrm{II}$ & GAAGATCTATCGACATCATGTCGTCGGCGA \\
\hline Sflef9C-NotI & GAATGCGGCCGCTCAATCCAAAAACATGTCTAACA \\
\hline Sflef10N-BglII & GTAGATCTTCTTCAGTGTCCATCGCGGAT \\
\hline Sflef10C-NotI & GAATGCGGCCGCTCATAGTGTTTGGTCACTTTGCA \\
\hline Sflef $11 \mathrm{~N}-B g l \mathrm{II}$ & GAAGATCTGATAAACAATCGTCGAGGCAGCA \\
\hline Sflef11C-NotI & GAATGCGGCCGCTCAGTTAAAGCGTTCGTTGTGGT \\
\hline Sfie1N-BamHI & CGGGATCCCACACTCTCAACGACAACGCCAA \\
\hline Sfie1C-NotI & GAATGCGGCCGCCTAACAGTTGTCGTTGTCCTGT \\
\hline SfdnapolN-BglII & GAAGATCTGTGGCGTTTTCGTTGCTCGA \\
\hline SfdnapolC-NotI & GAATGCGGCCGCTCAACAATCCCTTTCGCATTTGA \\
\hline Sfp143N-BglII & GAAGATCTGCGACTGCGGAGATAAGTGTTGA \\
\hline Sfp143C-Not I & GAATGCGGCCGCCTAACATATAAATTCAGGTTGATT \\
\hline Sf $p 47 \mathrm{~N}-$ Bam HI & CGGGATCCGGTTTTGCGCGTTTTTACGAGA \\
\hline Sf $p 47$ C-Not I & GAATGCGGCCGCTCAATATAATGTTACAAGTCTTTGA \\
\hline Sf39KN-BglII & GAAGATCTAACACCACAACGAACGCTTTAACT \\
\hline Sf39KC-NotI & GAATGCGGCCGCTTAACTATATGACATTTGTGTGT \\
\hline
\end{tabular}

CTTTGCTTTCCGCGAAACACTTTACCGAAAGCAAA GATCGAA- ${ }^{\prime}$ and $5^{\prime}$-CGCGTTCGATCTTTGCTTTCGG TAAAGTGTTTCGCGGAAAGCAAAGTTTGAA-3'

DNA transfections

Sf9 cells $\left(3.2 \times 10^{5}\right)$ were seeded in each well of 12-well plates and were transfected with the indicated amounts of DNA. Total DNA to be transfected per well was standardized by adding lambda DNA when needed, and mixed with $3 \mu \mathrm{l}$ of Cellfectin ${ }^{\circledR}$ (Invitrogen) reagent in serum free Sf900 II media. Cells were incubated at $27^{\circ} \mathrm{C}$ for $4 \mathrm{~h}$ and after that, the transfection mixture was replaced by fresh media containing $1 \%$ fetal bovine serum. The transfected cells were incubated at $27{ }^{\circ} \mathrm{C}$ for the period required for each assay.

Transient expression assays

For late gene expression assays, cells seeded in 12-well plates were transfected with $0.8 \mu \mathrm{g}$ of reporter plasmids and $0.2 \mu \mathrm{g}$ of each plasmid expressing the lefs that were included in each experiment. Cells were incubated at $27{ }^{\circ} \mathrm{C}$ for $48 \mathrm{~h}$ post-transfection. Luciferase activity was measured with Luciferase Assay System (Promega). In brief, cells were lysed for $5 \mathrm{~min}$ in $400 \mu \mathrm{l}$ of Reporter Lysis Buffer (Promega) at room temperature and then transferred into ice-chilled $1.5 \mathrm{ml}$ tubes, vortexed and 
centrifuged $30 \mathrm{~s}$ to pellet cell debris. Lysate $(20 \mu \mathrm{l})$ and Luciferase Assay Reagent ( $80 \mu \mathrm{l}$, Promega) were mixed in 96-well opaque plates and luminescence was measured with a Veritas ${ }^{\mathrm{TM}}$ Microplate Luminometer (Turner Biosystems), according to the manufacturer's instructions.

For early gene expression assays, cells seeded in each of 12-well plates were transfected with $1.0 \mu \mathrm{g}$ of reporter plasmids carrying the $p 35$ promoter elements and $0.5 \mu \mathrm{g}$ of plasmid expressing AcMNPV ie-1 or equimolar amounts of plasmids expressing SfMNPV ie-1. Cells were incubated at $27{ }^{\circ} \mathrm{C}$ for $24 \mathrm{~h}$ post-transfection and luciferase activity was measured as described for late gene expression assays.

When viral DNA was used in the assays, $0.5 \mu \mathrm{g}$ of the specified DNA was included in the transfection mix. All transfections were repeated in triplicate at different times, and the average of these experiments is presented.

\section{Immunoblotting}

To compare the levels of expression between AcMNPV $i e-1$ and SfMNPV ie- 1 cloned in vector $\mathrm{pHSEpiHisVI}^{+}$, the same cell extracts that were measured for luciferase activity were treated with $2 \times$ cracking buffer $(0.13 \mathrm{M}$ Tris pH 6.8, $4 \%$ sodium dodecyl sulfate [SDS], $20 \%$ glycerol, $0.14 \%$ bromphenol blue, $2 \%$ 2-mercaptoethanol) and boiled for $2 \mathrm{~min}$. Equal volumes of lysates were resolved by $12 \%$ SDS-polyacrylamide gel electrophoresis (PAGE). The resolved proteins were transferred to a nitrocellulose membrane (Whatman) and immunolabeled with 1:500 dilution of anti-HA monoclonal antibody (Invitrogen) and 1:1,000 dilution of goat anti-mouse IgG-HRP (horseradish peroxidase) (SIGMA) and detected by chemiluminescence with ECL western blot reactives (Pierce, Thermo Scientific).

\section{Results and discussion}

Transactivation of a late promoter by SfMNPV lefs and complementation by AcMNPV lefs

To construct the collection of clones we designated as SfMNPV lef library, we cloned individually all SfMNPV lefs listed in Table 1, fused to HA and His tags, under Drosophila hsp 70 promoter, in the expression vector pHSEpiHisVI $^{+}$[13] (see "Materials and methods" section for details). This vector was originally developed to express constitutively AcMNPV lefs in Sf 21 cells. The AcMNPV lef library (previously designated pHSEpiHis lef library [13]), including all AcMNPV lefs listed in Table 1, has been found to support expression of a reporter gene under control of the late promoter of AcMNPV vp39 capsid gene, in transient assays, in Sf 21 cells. In the present study, we adapted this system to assess the functionality of SfMNPV lefs to support late gene expression, in Sf9 cells. To this end, the complete SfMNPV lef library was cotransfected into cells along with a reporter plasmid containing AcMNPV vp39 promoter-driven luciferase, cislinked to an $h r$ fragment. This promoter was responsive to activation by both AcMNPV and SfMNPV, in the context of Sf9 cells transfected with viral DNA (Fig. 1b). This is consistent with the high level of sequence identity of $v p 39$ promoter regions of both viruses, including two conserved TAAG motifs proximal to the ATG translational start codon, probably due to overlap with the $5^{\prime}$ end of lef-4 gene (which is encoded in the complementary DNA strand). We tested $h r$ sequences from each virus in the reporter plasmid since, in transient assays, hrs have been shown to have species-specific effects on both virally-induced replication of plasmids [29, 34] and transactivation of a late gene promoter [35].

When the AcMNPV lef library was cotransfected with reporter plasmid pAchr5CAPluc, containing an AcMNPVderived $h r$ (Ac $h r$ ), maximum levels of reporter activity were obtained (Fig. 1a, column 3, positive control). The ability of the AcMNPV lef library to transactivate the late promoter was specific with regard to the $h r$ sequence; when an SfMNPV-derived $h r(\mathrm{Sf} h r)$ was present, as in reporter plasmid $\mathrm{pSf} h r l \mathrm{CAPluc}$, reporter activity dropped to about $4 \%$ of the positive control (Fig. 1a, column 4). Comparatively, the SfMNPV lef library cotransfected with pSf $h r l$ CAPluc resulted in $2 \%$ the activity of the positive control (Fig. 1a, column 6). Even though this value is very low, it is above background levels obtained with the SfMNPV lef library cotransfected with pAchr5CAPluc (Fig. 1a, column 5). It is worth to mention that $\mathrm{Sf} h r$ spans five palindromes of the $h r l$ region of SfMNPV, while Ac $h r$ contains only two palindromes from AcMNPV $h r 5$ (see "Materials and methods" section). In relation to the role of $h r s$ as enhancers of early genes, the strength of the transactivation correlates primarily with the number of palindromes in the $h r$ [36]. We do not know whether the $h r$ length could affect activation of the late promoter noticeably, in the assay. In any case, the lower activity obtained with the SfMNPV lef library compared to that obtained with the AcMNPV lef library does not correlate with the length of the $h r$ in the reporter plasmids.

In these experiments, AcMNPV p35 (see Table 1) was cotransfected with the SfMNPV lef library. There is evidence that the functional contribution of $p 35$ to the AcMNPV lef library is to prevent apoptosis of transfected cells [37, 38]. Therefore, since SfMNPV lacks a p35 homolog, the AcMNPV gene was included in both libraries to avoid artifacts that could arise due to cell viability. In parallel experiments conducted without $p 35$ in the transfection mix, the activity of the reporter obtained with the 

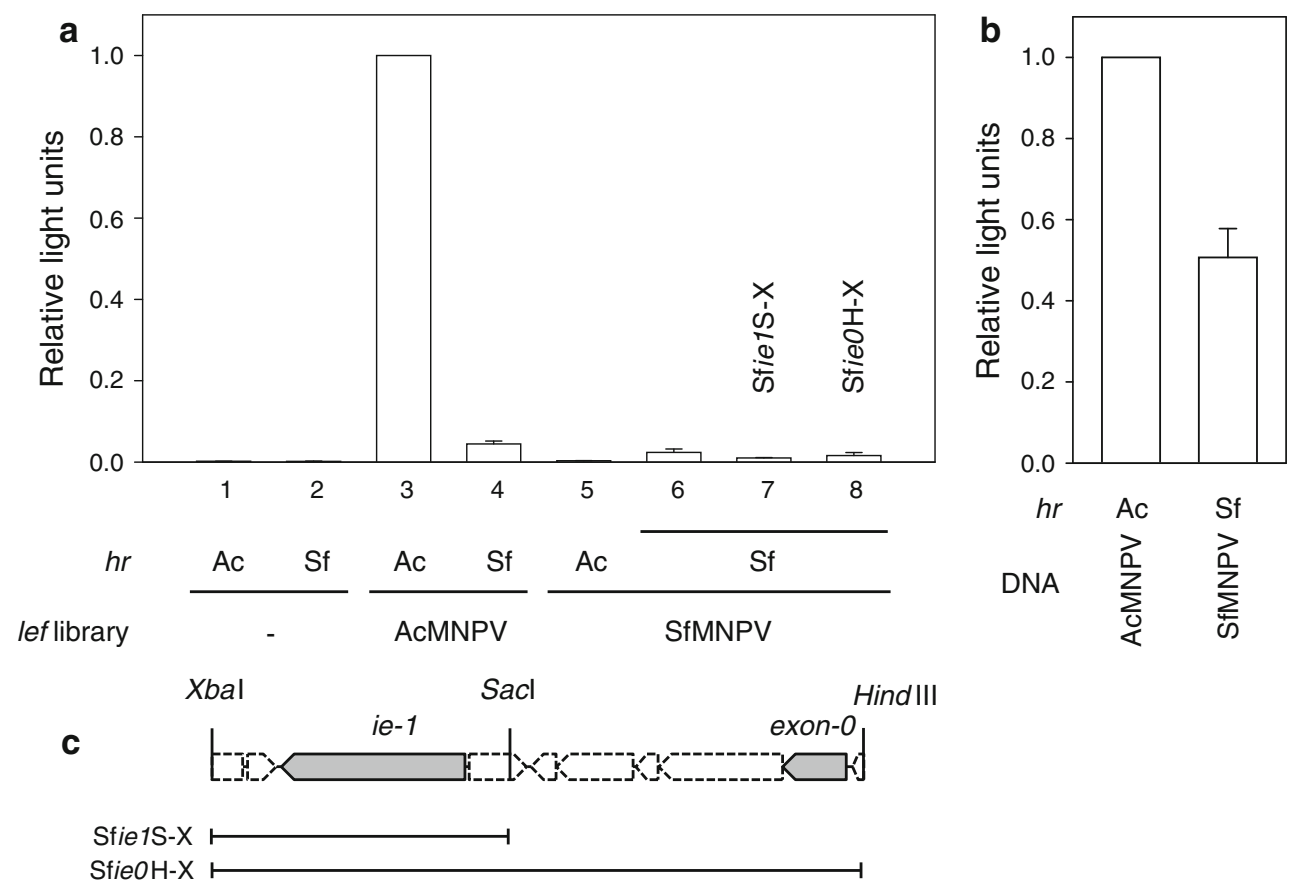

Fig. 1 Late gene expression in the presence of SfMNPV lefs and SfMNPV DNA in Sf9 cells. a Sf9 cells were cotransfected with AcMNPV or SfMNPV lef libraries $(0.2 \mu \mathrm{g}$ of each lef) and reporter plasmids $(0.8 \mu \mathrm{g})$ pAchr5CAPluc, containing a fragment of AcMNPV $h r 5$ (Ac $h r$ ), or pSfhrlCAPluc, containing a fragment of SfMNPV $h r l$ ( $\mathrm{Sf} h r$ ). SfMNPV ie-1 was replaced in SfMNPV lef library by genomic clones Sfie $1 \mathrm{~S}-\mathrm{X}$ (column 7) or SfieOH-X (column 8 ). Cells were harvested $48 \mathrm{~h}$ post-transfection and lysates were

SfMNPV lef library decreased by as much as $40 \%$ (data not shown).

Results presented here indicate that SfMNPV lefs, as a group, have very low late promoter transactivation capacity in the assay. The difference observed in reporter activation by AcMNPV and SfMNPV lef libraries is in contrast to more similar activities observed when genomic DNA of each virus was cotransfected with the indicated reporter plasmid (Fig. 1b). One possible explanation is that SfMNPV factors other than those represented in the lef library are required to stimulate late gene expression.

In a previous study, it was shown that IE-1 of Lymantria dispar MNPV (LdMNPV), other member of the group II alphabaculoviruses, did not support transient plasmid replication when expressed in Ld652Y cells along with the remaining LdMNPV replication factors. In contrast, IE-0, a variant of IE-1, was required to obtain replication of the reporter plasmid [39]. In species in which they were studied experimentally, IE- 1 differs from IE- 0 in that the latter has an extended $\mathrm{N}$-terminus, since it is translated from a spliced transcript that encodes portion of exon- 0 ORF in frame with ie-1 ORF [39-41]. Although these products are functionally similar they may exhibit differential properties in different contexts $[39,42,43]$. Since analyzed for luciferase activity. Luciferase activity from cells cotransfected with AcMNPV lef library and reporter plasmid pAchr5CAPluc (column 3) was set to 1.0. b Transactivation of the indicated reporter plasmids by cotransfection with AcMNPV or SfMNPV viral DNA $(0.5 \mu \mathrm{g})$. c Schematic diagram of the SfMNPV genomic region encompassing ie-1 and exon-0 ORFs (shaded arrows). Restriction fragments cloned in plasmids SfielS-X and SfieOH-X are indicated

replication of the reporter plasmid is necessary for activation of the late promoter in the assay, we assessed the complementing capacity of an SfMNPV genomic fragment from which ie-O could be expressed to explore possible requirement of an SfMNPV IE-0 homolog for promoter activation. Plasmid SfieOH-X (Fig. 1c) was constructed to contain a $7.2 \mathrm{kbp}$ HindIII-XbaI genomic fragment that spans from sequences upstream exon- 0 to downstream ie-1 ORFs. This fragment is homologous to regions that function as templates for synthesis of the spliced ie- 0 mRNA in other baculoviruses. In addition we tested plasmid SfielS-X, a subclone of SfieOH-X that represents a SacI$X b a \mathrm{I}$ genomic fragment containing only $i e-1$ along with a few hundred base pair flanking sequences. SfMNPV $i e-1$ in the lef library was replaced by each $i e-1$-containing genomic clone and the resulting libraries were cotransfected with reporter plasmid pSf $h r l C A P l u c$. Reporter activity did not increase in the presence of SfieOH-X in the library (Fig. 1a, column 8) compared to that with the original lef library. A similar result was obtained with SfielS-X (Fig. 1a, column 7). Since we did not confirm expression of IE-0 from SfieOH-X, these results preclude us from making definitive conclusions about IE-0 requirement in the assay. 
Besides $p 35$, two other AcMNPV lefs are not present in the genome of SfMNPV: $i e-2$ and lef-12 (see Table 1). ie-2 is considered stimulatory for DNA replication in a cellline-specific manner [17], while lef-12 is thought to have specific influence in transcription [44]. According to current knowledge, the remaining AcMNPV lefs are also predicted to function mainly through their involvement in either replication- or transcription-specific events. In the experiments presented below, we defined the replication group of lefs including: lef-1, lef-2, lef-3, lef-7, lef-11, ie-1, dnapol and p143; and the transcription group containing: lef-4, lef-5, lef-6, lef-8, lef-9, lef-10, p47, and 39K. To investigate a possible link between low reporter gene activity and lack of functionality of either subgroup of SfMNPV lefs, we replaced each subgroup of genes in the SfMNPV lef library with the corresponding subgroup of the AcMNPV lef library, and viceversa. AcMNPV ie-2 and lef-12 were assessed separately to evaluate their contribution in different contexts. Results shown in Fig. 2 indicate that there are two independent principal components of the assorted libraries that maximize reporter activity: AcMNPV replication lefs (Fig. 2, compare columns 1-4 to columns 5-8), and AcMNPV-specific lefs ie-2 and lef-12 (Fig. 2, compare odd columns to even columns). This implies that SfMNPV transcription lefs substituted for the corresponding AcMNPV lefs activity very efficiently, from 76 to $95 \%$, depending of the library context (Fig. 2, compare column $3-1$, and column $8-6$, respectively). Controls with only one subgroup of lefs of either library were omitted for simplicity, since they resulted in background levels of reporter gene activity.
In addition to the experiments presented here, we tested the combination of AcMNPV replication and SfMNPV transcription lefs, with or without ie-2/lef-12 (as indicated for columns 3 and 4 of Fig. 2) in cotransfections with the reporter plasmid pSfhrlCAPluc. Reporter activity resulted in 1.5 and $0.5 \%$ the activity of positive control, respectively (data not shown). These results suggest that the specificity of the interaction with the $h r$, in the context of the late promoter activation, is primarily restricted to the replication lefs.

AcMNPV $i e-2$ and lef-12 stressed the ability of SfMNPV lefs to support late promoter activity. They augmented reporter activity from 3- to 6-fold depending whether the library contained either AcMNPV or SfMNPV replication lefs, respectively (Fig. 2, e.g. compare column $3-4$, and column 5-6, respectively). ie-2 is missing in the whole lineage of group II alphabaculoviruses while lef-12 is present in some members of this group. It is not known whether SfMNPV encodes functional homologs of these genes.

In order to explore further the limitation posed by SfMNPV replication lefs to attain substantial activation of the late promoter, there are same questions that need to be addressed. For example, substitutions of individual lefs may lead to the identification of a specific lef being the limiting factor. Also, the dosage effect should be analyzed for lefs exhibiting low protein expression levels. In its current state, we proposed our system as a useful tool to systematically interrogate SfMNPV genome for genes able to mimic the stimulatory effect of $i e-2$ and lef- 12 on the SfMNPV lef library. Besides their fundamental role in the
Fig. 2 Late gene expression using combinations of subgroups of SfMNPV and AcMNPV lefs involved in transcription or replication. Sf9 cells were cotransfected with lef libraries containing different combinations of AcMNPV and SfMNPV lefs as indicated (+) and reporter plasmids pAchr5CAPluc (Ac $h r)$ or pSf $h r l$ CAPluc (Sf $h r)$. Replication (Rep) lefs include lef-1 to -3, lef-7, lef-11, ie-1, dnapol, and p143. Transcription (Tran) lefs include lef-4 to -6 , lef-8 to $-10,39 \mathrm{~K}$ and $p 47$. Cells were harvested $48 \mathrm{~h}$ posttransfection and lysates were analyzed for luciferase activity. Luciferase activity from cells cotransfected with the entire AcMNPV lef library and reporter plasmid pAchr5CAPluc (column 1) was set to 1.0

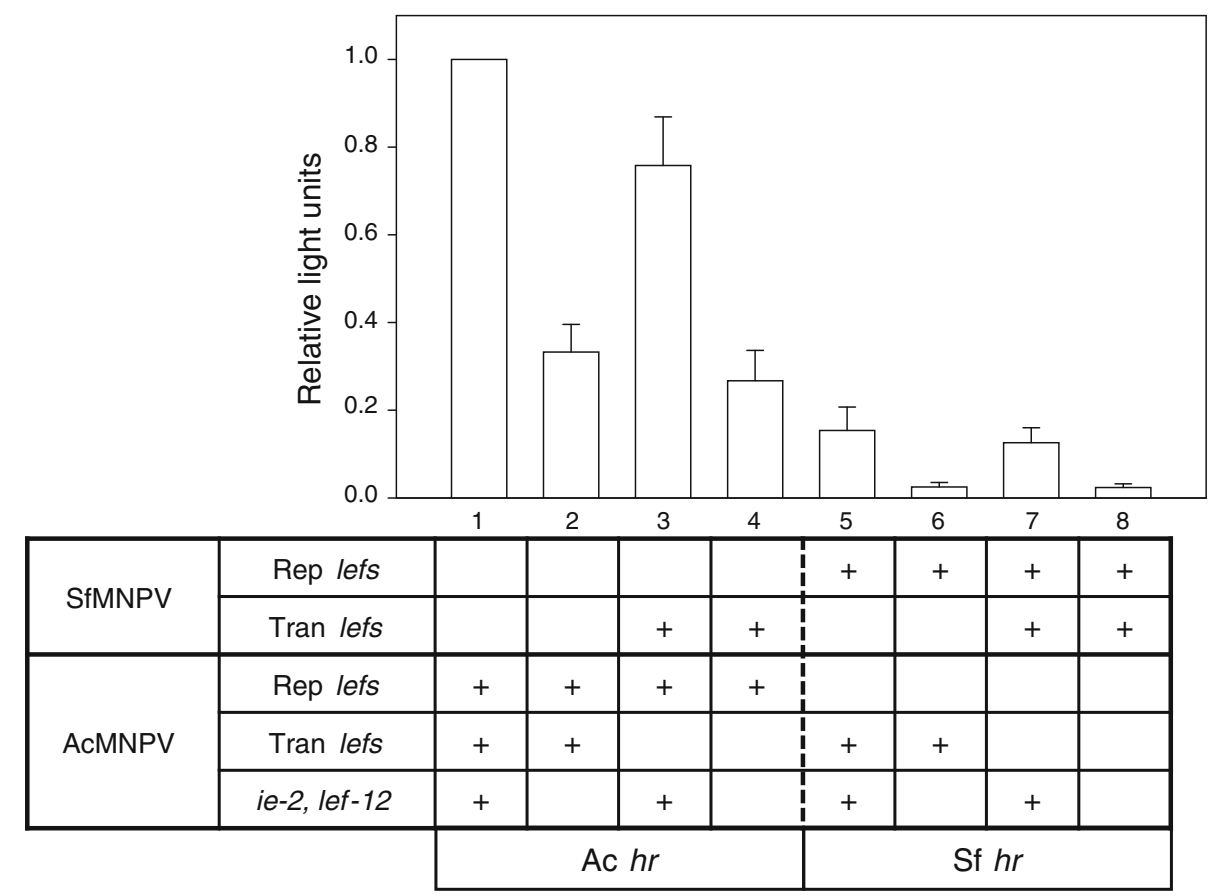


infection cycle, some lefs have been involved in determining host range in AcMNPV. To this regard, AcMNPV is considered a generalist virus. Therefore, the identification of possible new lefs in a specialist virus like SfMNPV, which is also a representative of a different group of alphabaculoviruses compared to AcMNPV, may contribute to new findings in relation to this trait.

Transactivation of an early promoter cis-linked to an AcMNPV- or SfMNPV-derived $h r$ element by SfMNPV ie-1-containing clones

IE-1 is involved in multiple processes during the virus infection cycle and may exhibit differential properties, including possible differential roles for IE-1 and IE-0 in each process. IE-1 functions have not been studied extensively among members of group II of alphabaculoviruses.
As mentioned above, IE-1 of LdMNPV was not active for plasmid replication in Ld652Y cells. Moreover, only IE-0 supported expression from an early promoter in both Ld652Y and Sf9 cell-lines [39]. In contrast, IE-1 of Spodoptera exigua MNPV (SeMNPV) was functional in a transient early gene expression assay, in Sf21 cells [35]. Since low reporter activity supported by SfMNPV lefs in late expression assays was mainly associated with the group of replication lefs, in which $i e-1$ is included, we investigated the ability of SfMNPV IE-1 to stimulate early gene expression in a model system. To drive the reporter gene we used the basal portion of the well characterized AcMNPV p35 early promoter, encompassing the TATA box motif and the transcription initiation site, cis-linked to an $h r$ element. In previous studies this promoter motifs, linked to an AcMNPV $h r$-derived single palindromic repeat (Ac 28-mer), were found to be the minimal elements
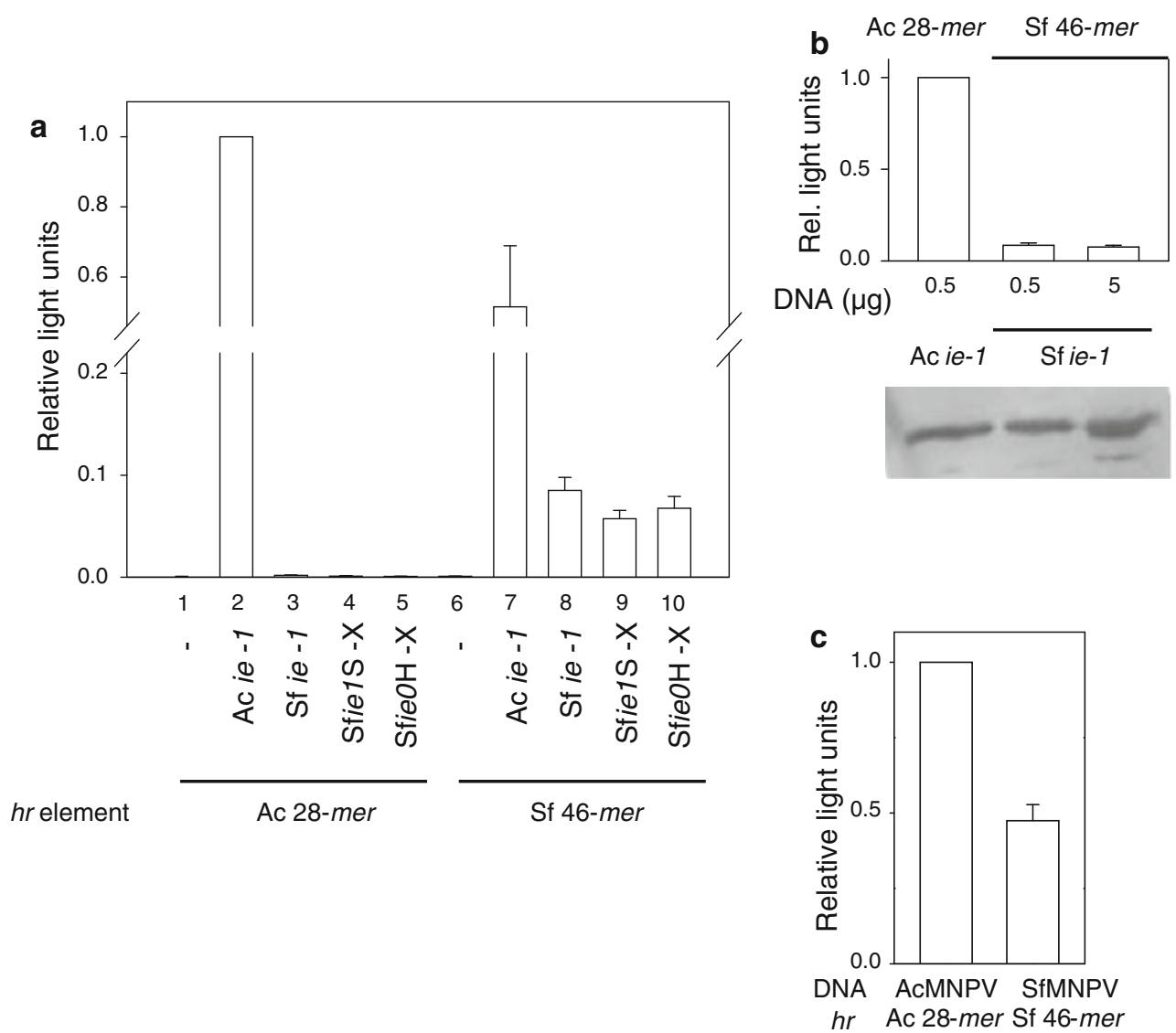

Fig. 3 Transactivation of the early $p 35$ basal promoter by SfMNPV IE-1. a Sf9 cells were cotransfected with $0.5 \mu \mathrm{g}$ of AcMNPV ie-1 (Ac $i e-1$ ), SfMNPV ie-1 (Sf ie-1) or equimolar amounts of plasmids Sfie1S-X or SfieOH-X, and reporter plasmids $(1.0 \mu \mathrm{g})$ pBAS35 $\mathrm{K}$-Luc/28mer-up+/PA (Ac 28-mer) or pBAS35K-Luc/46mer (Sf 46-mer). Reporter plasmids contained the luciferase gene under control of the p35 basal promoter, which encompasses the TATA element and RNA start site, cis-linked to a palindromic 28-mer from AcMNPV $h r 5$ or a palindromic 46-mer from SfMNPV $h r l$, respectively. Ac $i e-1$ and $\mathrm{Sf} i e-1$ express the corresponding IE-1 products fused to HA and His tags, under control of hsp 70 promoter. Cells were harvested $24 \mathrm{~h}$ post-transfection, and lysates were analyzed for luciferase activity. Luciferase activity from cells cotransfected with Ac ie-1 and reporter Ac 28-mer (column 2) was set to 1.0. b Sf ie-1 was tested as in panel "a" but using the indicated amount of DNA in transfections. Expression was tested using an aliquot of a lysate also used to measure luciferase activity and is shown below each column. c Transactivation of the indicated reporter plasmid by cotransfection with AcMNPV or SfMNPV viral DNA $(0.5 \mu \mathrm{g})$ 
required for $h r$-enhanced activation mediated by AcMNPV IE-1 [33]. This construct was also responsive to activation by heterologous IE-1 when Ac 28-mer was replaced by an $h r$ element from the same viral species as the IE-1 protein [35]. Therefore, in the present study, we used reporter plasmids containing luciferase under control of the $p 35$ basal promoter with either Ac 28-mer or an SfMNPV $h r$ derived palindromic repeat ( $\mathrm{Sf}$ 46-mer) [27] inserted upstream of the promoter. Cotransfection of SfMNPV viral DNA and reporter plasmid having Sf 46-mer into Sf9 cells stimulated expression from reporter gene though the efficiency was about $50 \%$ of that obtained by cotransfection of AcMNPV DNA and Ac 28-mer-containing plasmid (Fig. 3c).

To test promoter activation by $i e-1$, we cotransfected Sf9 cells with reporter plasmids and $i e-1$-expressing clones from the AcMNPV and SfMNPV lef libraries, or the SfMNPV genomic fragments tested in late expression assays (see above). AcMNPV ie- 1 supported the highest level of reporter activity in the presence of the reporter plasmid with Ac 28-mer (Fig. 3a, column 2, positive control). In the presence of Sf 46-mer in the reporter plasmid, AcMNPV ie-1 resulted in $51 \%$ reporter activity compared to that of positive control (Fig. 3a, column 7). SfMNPV ie-1 consistently enhanced expression of the reporter gene in plasmid with Sf 46-mer (Fig. 3a, column 8); nevertheless, the level of expression represented at most about $10 \%$ of the positive control. There were no significant differences in activity from this reporter when cotransfected with plasmid SfieOH-X, which is predicted to express IE-0 (Fig. 3a, column 10). Therefore, we could not propose a preferential role to either IE- product in the activation of the promoter, in our assay. SfMNPV $i e-1$ clones failed to stimulate reporter activity from plasmid containing Ac 28-mer (Fig. 3a, columns 3-5). This may reflect more strict constraints for the specificity of interaction between SfMNPV IE-1 and $h r$ elements in comparison to AcMNPV IE-1. The levels of expression from HA-tagged AcMNPV $i e-1$ and SfMNPV ie-1 were similar as revealed by immunoblotting (Fig. 3b); therefore, lower transcriptional activation observed with SfMNPV ie-1 was not a consequence of lower amount of protein. In fact, transfection with higher concentration of SfMNPV ie-1 DNA did not produce an increase in reporter activity.

In comparison, reporter activity obtained with SfMNPV DNA relative to that obtained with AcMNPV DNA was higher than the relative activity of the reporters obtained with the corresponding ie- 1 clones. This suggests that in the context of the viral infection SfMNPV IE-1 may be better expressed or may be the target of modifications that are needed to upregulate the activity of the protein product. This question could be addressed by testing clones of a genomic library of the virus to identify genes influencing positively
IE- 1 activity as a transactivator of the early promoter in the assay. Alternatively, SfMNPV IE-1 may have a more strict dependence to a native $h r$ sequence than AcMNPV IE-1, in order to mediate activation of an early promoter. Native $h r s$ have sequences between palindromes that bind cellular transcription factors which cooperate with IE-1-mediated transactivation of early promoters. In support of this, linkage to native $h r$ sequences activates early promoters to some extent in insect cells, even in the absence of IE-1 [45].

Acknowledgments The authors thank Dr. A. Lorena Passarelli for providing the AcMNPV lef library, plasmids pBAS35K-Luc/28mer$\mathrm{up}+/ \mathrm{PA}$ and pAchr5CAPluc, and for critically reading the manuscript. We are also grateful to O. Marcelo Farinón for excellent technical assistance. This study was supported by grants from the National Institute for Agricultural Technology (INTA) and the Agencia Nacional de Promoción Científica y Tecnológica (ANPCyT). MFB, OT, and VR hold research career awards from Consejo Nacional de Investigaciones Científicas y Técnicas (CONICET).

\section{References}

1. E.B. Carstens, L.A. Ball, Arch. Virol. 154, 1181-1188 (2009)

2. S.A.B. Miele, M.J. Garavaglia, M.N. Belaich, P.D. Ghiringhelli, Int. J. Evol. Biol. (2011). doi:10.4061/2011/379424

3. P.M. Zanotto, B.D. Kessing, J.E. Maruniak, J. Invertebr. Pathol. 62, 147-164 (1993)

4. P.D. Friesen, in The Baculoviruses, ed. by L.K. Miller (Plenum Press, New York, 1997), p. 141

5. S.M. Rodems, P.D. Friesen, J. Virol. 69, 5368-5375 (1995)

6. M.N. Pearson, R.M. Bjornson, G.D. Pearson, G.F. Rohrmann, Science 257, 1382-1384 (1992)

7. M. Kool, J.T.M. Voeten, R.W. Goldbach, J. Tramper, J.M. Vlak, J. Gen. Virol. 74, 2661-2668 (1993)

8. C. Rankin, B.G. Ooi, L.K. Miller, Gene 70, 39-49 (1988)

9. N.E. Huh, R.F. Weaver, J. Gen. Virol. 71, 195-202 (1990)

10. L.A. Guarino, B. Xu, J. Jin, W. Dong, J. Virol. 72, 7985-7991 (1998)

11. A.L. Passarelli, L.A. Guarino, Curr. Drug Targets 8, 1103-1115 (2007)

12. A.L. Passarelli, L.K. Miller, J. Virol. 67, 2149-2158 (1993)

13. J.C. Rapp, J.A. Wilson, L.K. Miller, J. Virol. 72, 10197-10206 (1998)

14. M. Kool, C. Ahrens, R.W. Goldbach, G.F. Rohrmann, J.M. Vlak, Proc. Natl Acad. Sci. U.S.A. 91, 11212-11216 (1994)

15. A. Lu, L.K. Miller, J. Virol. 69, 975-982 (1995)

16. S. Maeda, S.G. Kamita, A. Kondo, J. Virol. 67, 6234-6238 (1993)

17. A. Lu, L.K. Miller, J. Virol. 69, 6265-6272 (1995)

18. G.F. Rohrmann, Baculovirus Molecular Biology, 2nd edn. (Bethesda: National Center for Biotechnology Information, 2011), http://www.ncbi.nlm.nih.gov/books/NBK49500/

19. T. Williams, D. Goulson, P. Caballero, J. Cisneros, A.M. Martínez, J.W. Chapman, D.X. Roman, R.D. Cave, Biol. Control 14, 67-75 (1999)

20. R.W. Behle, H.J.R. Popham, J. Invertebr. Pathol. 109, 194-200 (2012)

21. A.H. McIntosh, C.M. Ignoffo, P.L. Andrews, Intervirology 23, 150-156 (1985)

22. G.M. Danyluk, J.E. Maruniak, J. Invertebr. Pathol. 50, 207-212 (1987)

23. H.-S. Liu, S.L. Bilimoria, Arch. Virol. 115, 101-113 (1990) 
24. M.R. Pedrini, J.L. Caldas Wolff, S. Reid, Ann. Appl. Biol. 145, 107-112 (2004)

25. H.-S. Liu, S.L. Bilimoria, Intervirology 40, 50-54 (1997)

26. J.L. Caldas Wolff, F.H. Valicente, R. Martins, J.V. Oliveira, P.M. Zanotto, J. Gen. Virol. 89, 1202-1211 (2008)

27. R.L. Harrison, B. Puttler, H.J.R. Popham, J. Gen. Virol. 89, 775-790 (2008)

28. O. Simón, L. Palma, I. Beperet, D. Muñoz, M. López-Ferber, P. Caballero, T. Williams, J. Invertebr. Pathol. 107, 33-42 (2011)

29. J.G.M. Heldens, Y. Liu, D. Zuidema, R.W. Goldbach, J.M. Vlak, J. Gen. Virol. 78, 3101-3114 (1997)

30. M.N. Pearson, G.F. Rohrmann, J. Virol. 72, 9142-9149 (1998)

31. M.F. Berretta, M.L. Ríos, A. Sciocco de Cap, J. Invertebr. Pathol. 71, 280-282 (1998)

32. M.F. Berretta, M. Deshpande, E.A. Crouch, A.L. Passarelli, Virology 348, 175-189 (2006)

33. V.A. Olson, J.A. Wetter, P.D. Friesen, J. Virol. 75, 6042-6051 (2001)
34. R. Broer, J.G.M. Heldens, E.A. van Strien, D. Zuidema, J.M. Vlak, J. Gen. Virol. 79, 1563-1572 (1998)

35. M.F. Berretta, A.L. Passarelli, Virology 355, 82-93 (2006)

36. S.M. Rodems, P.D. Friesen, J. Virol. 67, 5776-5785 (1993)

37. J. Todd, A.L. Passarelli, L.K. Miller, J. Virol. 69, 968-974 (1995)

38. E.A. Prikhod'ko, L.K. Miller, J. Virol. 70, 7116-7124 (1996)

39. M.N. Pearson, G.F. Rohrmann, Virology 235, 153-165 (1997)

40. G.E. Chisholm, D.J. Henner, J. Virol. 62, 3193-3200 (1988)

41. D.A. Theilmann, L.G. Willis, B.J. Bosch, I.J. Forsythe, Q. Li, Virology 290, 211-223 (2001)

42. I. Huijskens, L. Li, L.G. Willis, D.A. Theilmann, Virology 323, 120-130 (2004)

43. T.M. Stewart, I. Huijskens, L.G. Willis, D.A. Theilmann, J. Virol. 79, 4619-4629 (2005)

44. L.A. Guarino, T.A. Mistretta, W. Dong, J. Virol. 76, 1203212043 (2002)

45. I. Landais, R. Vincent, M. Bouton, G. Devauchelle, M. DuonorCerutti, M. Ogliastro, Virology 344, 421-431 (2006) 